\title{
Curva J. Controversias
}

\section{J curve: controversies}

\author{
Edwin Rolando Castillo-Velarde ${ }^{1,2}$ \\ ${ }^{1}$ Nefrólogo, Hospital Nacional Guillermo Almenara Yrigoyen, EsSalud. \\ ${ }^{2}$ Miembro de la Sociedad Peruana de Hipertensión Arterial.
}

\begin{abstract}
Resumen
Para el logro de un óptimo control de la hipertensión arterial, además de entender adecuadamente su base fisiopatológica, clínica, terapéutica, se requiere conocer el sustento que poseen las recomendaciones sobre los objetivos de tratamiento, así como los beneficios y riesgos del mismo. En este sentido, una consecuencia descrita asociada al manejo antihipertensivo es la presencia de la curva J, es decir la presencia de un aumento de riesgo cardiovascular asociado a la reducción de la presión diastólica. Si bien esta curva ha sido debatida por muchos años, existen múltiples reportes sobre su existencia y su impacto sobre la morbimortalidad, por lo cual constituye una referencia importante en el momento de establecer nuestros objetivos de tratamiento.

Palabras clave: Enfermedades cardiovasculares; presión sanguínea; curva J.
\end{abstract}

\begin{abstract}
Optimal control of hypertension requires knowledge of the pathophysiologic, clinical and therapeutic mechanisms, as well as support of recommendations on treatment goals, benefits and risks. Consequence of antihypertensive management is the presence of the J-curve that represents the presence of increased cardiovascular risk associated with diastolic pressure reduction. Although this curve has been debated for years, there are numerous reports about its existence and impact on morbidity and mortality, thereby providing an important reference when setting our goals of treatment.
\end{abstract}

Key words: Cardiovascular diseases; blood pressure; I curve.
Es un hecho abiertamente demostrado que un adecuado control de la presión arterial reduce la morbimortalidad de los pacientes con hipertensión arterial. El séptimo reporte del Joint Nacional Committee $^{(1)}$, vigente desde el 2003, considera una presión arterial normal como aquella PAS $<120 \mathrm{mmHg}$ y $\mathrm{PAD}<80 \mathrm{mmHg}$, prediciendo una duplicación del riesgo de mortalidad por cada aumento de 20 $\mathrm{mmHg}$ de presión sistólica o $10 \mathrm{mmHg}$ de presión diastolica. Por otro lado, las guías europeas estiman como presión arterial normal aquella PAS de 120 a $129 \mathrm{mmHg}$ y PAD de 80 a $84 \mathrm{mmHg}$. Al considerar grupos específicos de tratamiento, existen recomendaciones para presiones objetivo, como $<130 / 80 \mathrm{mmHg}$ en pacientes de riesgo alto -como en diabetes mellitus o enfermedad renal crónica-y una presión de 125/75 mmHg en pacientes nefrópatas con proteinuria $>1 \mathrm{~g} /$ día. Bajo estos lineamientos, la Sociedad Británica de Hipertensión ${ }^{(2)}$, en 2004, recomendó una política de 'menor es mejor', para el manejo óptimo de la presión arterial.

Sin embargo, existe otra consideración. Stewart ${ }^{(3)}$, en 1979 , sugirió que una reducción excesiva de la presión sanguínea diastólica relacionada al tratamiento antihipertensivo se asociaba a un aumento de muertes por enfermedad arterial coronaria. Es así que se empezó a comunicar la presencia de una curva J, la cual ha sido materia de debate por muchos anos.

El fenómeno de curva J se define como el riesgo no linear de incremento de eventos cardiovasculares [enfermedad coronaria, apoplejía (stroke) y falla cardiaca] asociados a una reducción de la presión diastólica ${ }^{(4)}$, siendo últimamente esta asociación mayormente vinculada a la enfermedad coronaria. Si bien no hay duda de que existe un punto J a partir del cual se compromete la perfusión adecuada de órganos vitales, la esencia del debate recae en que si este fenómeno existe en un rango de presión arterial relacionado al tratamiento antihipertensivo.

Se ha planteado 3 explicaciones de la curva J:

- Es un fenómeno observado en pacientes ancianos con hipertensión sistólica aislada asociado a incremento de la presión de pulso, que a su vez se relaciona a incremento del riesgo cardiovascular.

- Es un epifenómeno asociado a diversas enfermedades, principalmente a disfunción cardiaca. Esta explicación se relaciona a una 'causalidad reversa'.
- Es observado en aquellos pacientes con una reserva coronaria disminuida que, al disminuir la presión diastólica como consecuencia del tratamiento antihipertensivo, desencadena un evento coronario.

\section{CURVA J ASOCIADA A AUMENTO DE LA RIGIDEZ ARTERIAL}

La presión de pulso (PP, definido como la diferencia entre la presión sistólica y diastólica) es un fenómeno observado progresivamente con la edad, en donde el radio de $\mathrm{PD} / \mathrm{PS}$ va declinando en el tiempo, siendo más prevalente en la población anciana. En esta población, constituye un predictor de enfermedad cardiovascular más sensible que la presión sistólica, lo cual lo diferencia de la presión diastólica, que constituye más bien un predictor más sensible de enfermedad cardiovascular en la población menor de 50 años ${ }^{(4,5)}$.

Estrictamente en esta asociación, se atribuye un aumento de riesgo cardiovascular cuando se describe un aumento de la presión de pulso, en ausencia de evento cardiovascular previo, y no solo por una reducción de la presión diastólica. Esto se puede apreciar en el estudio de Framingham, en donde se identificó 
un mayor riesgo en aquellos con una PP>45 mmHg y una PAD $<80 \mathrm{mmHg}{ }^{(6)}$. Asimismo, este fenómeno sería posible identificarlo en personas con hipertensión sistólica aislada, en donde, según registro NHANES ${ }^{(7)}$ de 1999-2006, hasta $46 \%$ de las no tratadas y hasta $41 \%$ de las tratadas tienen una presión diastólica menor de $70 \mathrm{mmHg}$.

\section{CURVA J Y CAUSALIDAD REVERSA}

Esta explicación relaciona la curva J como un epifenómeno secundario a diversas enfermedades, que puede incluir aquellas con disfunción cardiaca. Se habla de causalidad reversa cuando la enfermedad (como infarto de miocardio) reduce el factor de riesgo primario (en este caso, la presión diastólica o sistólica). Lo cual muestra una relación entre disfunción cardiaca y curva J sistólica o diastólica, como se puede observar en el estudio VALUE ${ }^{(8)}$, en donde luego de un infarto de miocardio, aquellos pacientes con una PS sostenida $<100 \mathrm{mmHg}$ en los siguientes meses de control (mes 1,3 y 6 ) tuvieron peores eventos cardiovasculares, lo cual a su vez fue más frecuente en aquellos con mayor incidencia de ondas Q. Es por esta razón que, diversos estudios que han evaluado el impacto de la presencia de la curva J no consideran aquellos que han incluido a pacientes con infarto de miocardio. Otras enfermedades que se vinculan con una disminución de la presión sanguínea incluyen a neoplasias y malnutrición. Sin embargo, se ha seguido observando la presencia de curva J y mortalidad cardiovascular en estos pacientes luego de haber corregido estas variables por análisis de regresión ${ }^{(4,5,9)}$.

\section{CURVA J, RESERVA CORONARIA Y TERAPIA ANTIHIPERTENSIVA}

Esta explicación tendría una mayor evidencia, según explicaremos luego. El corazón, al recibir su aporte sanguíneo durante la diastole, es vulnerable a la isquemia en el contexto de una enfermedad arterial coronaria asociado a una terapia antihipertensiva. En pacientes con hipertrofia miocárdica izquierda, la isquemia subendocárdica puede ocurrir incluso en ausencia de estenosis. En la figura 1, apre- ciamos que estos pacientes poseen una menor capacidad de autorregulación, en especial a nivel subendocárdico ${ }^{(10)}$. Asimismo, existen diversos estudios que han demostrado precisamente la presencia de curva J en pacientes que fueron incluidos con enfermedad cardiovascular, previo a la aleatorizacion ${ }^{(11)}$.

\section{CURVA J. EVIDENCIA}

En los fines de 1990, se consideró que el debate de la curva J se había resuelto en relación a un metaanálisis publicado de más de 420000 pacientes, en el que se concluía la presencia de una curva linear entre presión arterial y riesgo cardiovascular, considerando incluso un menor riesgo de enfermedad cardiaca coronaria si se tenía una PAD $<69 \mathrm{mmHg}{ }^{(12)}$. Sin embargo, se realizaron observaciones importantes a este metanálisis, en razón a que, pese a su observación prospectiva de 10 años, sus conclusiones se basaron en la PD basal, no considerando la de seguimiento al tratamiento ${ }^{(11)}$. Considerando estas críticas y luego de la publicación de estudios que enfatizaban o no la presencia de la curva J, el debate reapareció ${ }^{(13)}$.

El estudio SHEP fue un estudio aleatorio, controlado por placebo, doble ciego, prospectivo, con seguimiento de 5 años, en 4362 pacientes, que tuvo como objetivo evaluar el impacto del tratamiento antihipertensivo en la morbimortalidad cardiovascular en una población mayor de 60 años con hipertensión sistólica aislada (PAS $\geq 160 \mathrm{mmHg}$ y PAD $<90$ $\mathrm{mmHg}$ ). Concluyó que una reducción de la presión arterial se asociaba a menor

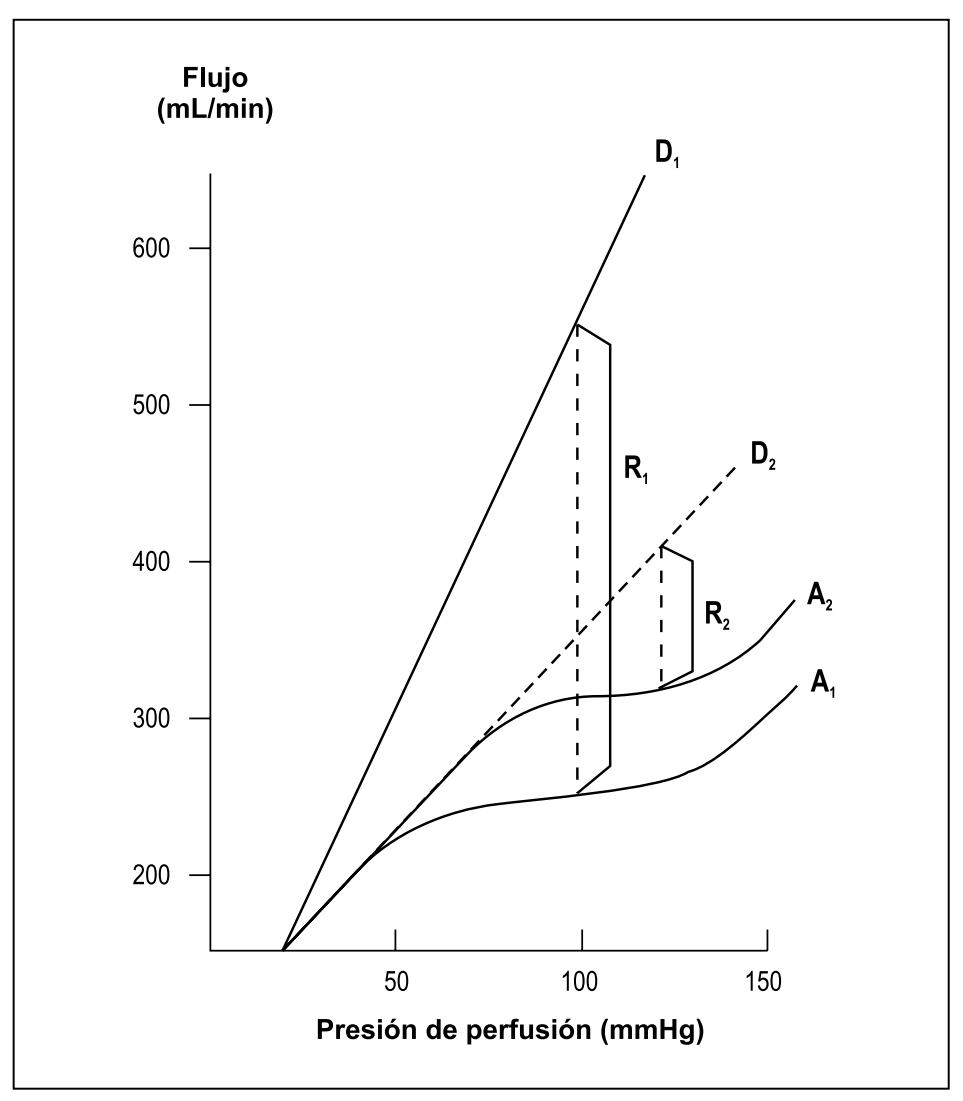

Figura 1. Autorregulación de flujo coronario en pacientes hipertensos con hipertrofia ventricular izquierda $(\mathrm{HVI})$. La tasa de extracción de oxígeno se incrementa con una presión de perfusión de $80 \mathrm{mmHg}$, se hace significativa con una presión de perfusión de 70 a $75 \mathrm{mmHg}$ y cesa cuando es menor de 40 a $50 \mathrm{mmHg}$. Se aprecia un ventrículo izquierdo (VI) normal (A1) durante un proceso de autorregulación con máxima vasodilatación (D1) y un ventrículo izquierdo hipertrofiado (A2), durante un proceso de autorregulación con máxima vasodilatación (D2). Existirá una menor reserva coronaria en la HVI (R2), en comparación del VI normal (R1). 


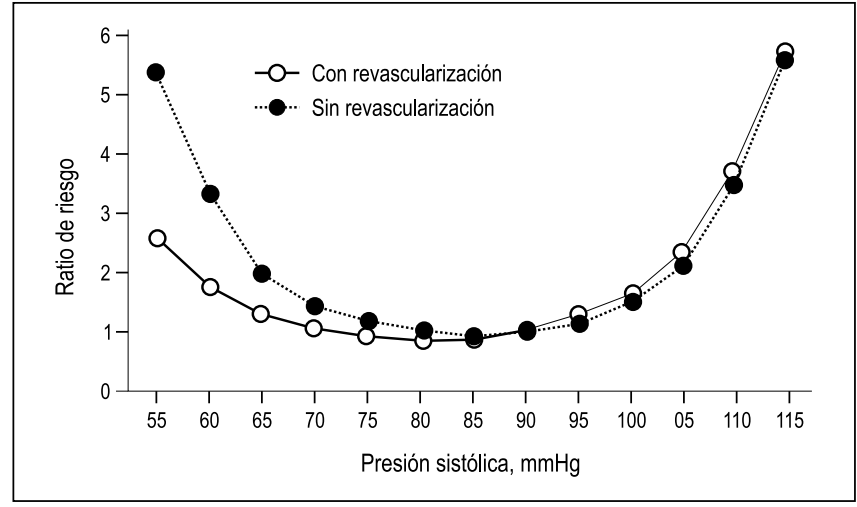

Figura 2. Derecha. Se aprecia la incidencia de eventos primarios (muerte total, infarto de miocardio no fatal y stroke no fatal) para diferentes presiones sistólica y diastólica. Superior. Se nota la interacción entre presión diastólica y evento primario en pacientes sometidos previamente o no a revascularización.

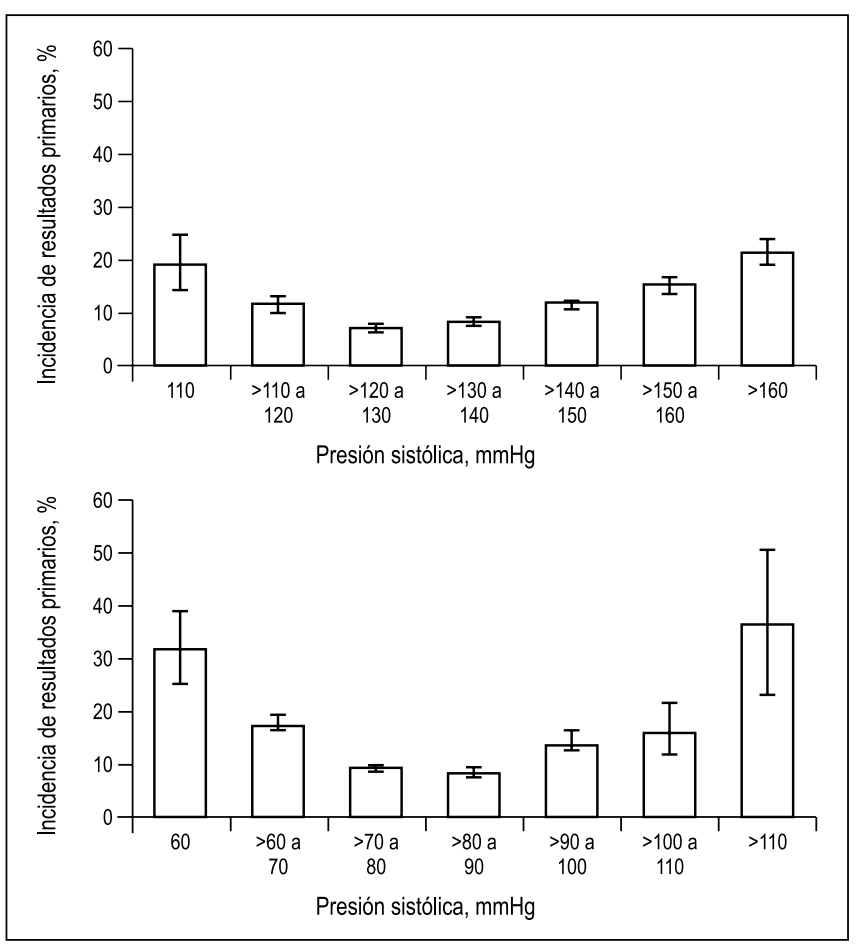

morbimortalidad cardiovascular. En un análisis posterior $^{(14)}$, se analizó la relación de la PP con enfermedad cardiaca coronaria, falla cardiaca y apoplejía (stroke). Se excluyó aquellos pacientes con historia de infarto de miocardio. Se describió una PP basal $>70 \mathrm{mmHg}(93,5 \mathrm{mmHg}$ en promedio), y solo $8 \%$ en el grupo tratamiento y $1 \%$ en el grupo placebo tenían PP <60 $\mathrm{mmHg}$. Se estimó que un incremento en $10 \mathrm{mmHg}$ de PP se asociaba al aumento de riesgo de falla cardiaca y stroke. Aquellos pacientes en el grupo de tratamiento y con $\mathrm{PD}<55 \mathrm{mmHg}$ no tuvieron mayor riesgo, pero en comparación al grupo placebo. En cambio, los pacientes en el grupo de tratamiento con PD $>55 \mathrm{mmHg}$ fueron significativamente mejor que el grupo placebo. Tal es así que una $\mathrm{PD}<55$ mmHg incrementó el riesgo en 2 veces en comparación a una PD de $70 \mathrm{mmHg}$. Asimismo, una reducción de la PD en $5 \mathrm{mmHg}$ aumentó en 14\% el riesgo de stroke. Esta reducción de PD e incremento de eventos cardiovasculares no estuvo en relación a otras variables, como cáncer, que se puede observar en mayor prevalencia en esta población ${ }^{(15)}$.

En 2002 se publicó un metaanálisis que evaluaba 1 millón de pacientes de 62 estudios prospectivos. En su diseño se excluyó aquellos pacientes con enfermedad cardiovascular previa, con análisis de la presión sistólica y diastólica durante su seguimiento, y no solo con valores basales. Este estudio concluyó que existía una relación lineal de reducción de mortalidad cardiovascular y reducción de la presión arterial, hasta valores de PAS de $115 \mathrm{mmHg}$ y PAD de $75 \mathrm{mmHg}$; sin embargo, por debajo de estos valores no existía suficiente evidencia para emitir conclusiones ${ }^{(16)}$.

Otros hallazgos interesantes fueron encontrados en un análisis secundario del estudio INVEST, el cual fue un estudio aleatorio, que evaluó más de 22000 pacientes con enfermedad cardiovascular e hipertensión, y que en su sub-estudio evaluaba el riesgo de eventos cardiovasculares asociado a una menor $\mathrm{PD}^{(9)}$. Se excluyó aquellos pacientes con infarto de miocardio y falla cardiaca 3 y 4 . En sus conclusiones, se identificó una curva J con un punto nadir de 119/84 mmHg. Este riesgo se duplicaba si se tenía una PD $<70 \mathrm{mmHg}$ y no se atribuyó solo a un aumento de la PP, ya que como consecuencia del tratamiento, se redujo ambas presiones sistólica y diastólica. Además, se describió un mayor riesgo de infarto de miocardio en relación a stroke.
Asimismo, aquellos pacientes a quienes se les realizó una revascularización coronaria toleraban mejor una menor presión diastólica, por lo que se vinculó esta curva J en aquellos pacientes con enfermedad cardiaca isquémica, como se aprecia en la figura 2 .

En razón a este vínculo con enfermedad cardiaca isquémica, se requería precisar la presencia de la curva J y eventos coronarios. Para ello se necesitaba de un estudio grande que comparara a pacientes hipertensos con cardiopatía isquémica y no isquémica con diferentes niveles objetivo de presión diastólica. Estos datos se pueden encontrar en el estudio HOT ${ }^{(17)}$. Este estudio incluyó a 19000 pacientes de 26 centros, el cual evaluaba la morbimortalidad cardiovascular en relación a tres niveles objetivo de presión diastólica (PD $\leq 90, \mathrm{PD} \leq 85, \mathrm{PD} \leq 80 \mathrm{mmHg}$ ). En sus conclusiones iniciales, manifestaron que una reducción de la PD en $82 \mathrm{mmHg}$ fue el punto más bajo para eventos cardiovasculares, y presiones por debajo de este nivel no se asociaron con incremento del riesgo cardiovascular, por lo que fue recomendada como 'segura'. Los pacientes diabéticos en especial se beneficiaron, si se encontraban en el grupo de $\mathrm{PD}<80$ $\mathrm{mmHg}$, en donde tuvieron una reducción 
Tabla 1. Infarto de miocardio fatal y no fatal en pacientes con y sin enfermedad cardiovascular isquémica (ECI).

\begin{tabular}{|c|c|c|c|c|c|c|c|c|c|c|}
\hline \multirow[b]{2}{*}{ Grupo } & \multirow[b]{2}{*}{$\begin{array}{l}\text { Objetivo } \\
\text { PD }\end{array}$} & \multirow[b]{2}{*}{$\mathrm{n}$} & \multirow[b]{2}{*}{$\begin{array}{l}\text { PS basal/ } \\
\text { PD }\end{array}$} & \multirow[b]{2}{*}{$\begin{array}{l}\text { PS/PD } \\
\text { obtenidas }\end{array}$} & \multicolumn{3}{|c|}{ Todos los IM + IM silentes } & \multicolumn{3}{|c|}{ Mortalidad por IM } \\
\hline & & & & & $\begin{array}{l}\text { Eventos } / 1000 \\
\text { pac. años }\end{array}$ & Comparación & $\overline{\mathrm{RR}}$ & $\begin{array}{l}\text { Eventos/1000 } \\
\text { pac. años }\end{array}$ & Comparación & $\mathrm{RR}$ \\
\hline \multirow[t]{3}{*}{$\operatorname{Sin} \mathrm{ECl}$} & $\leq 90$ & 5245 & $169 / 105$ & $143 / 85$ & 4,7 & 90 vs. 85 & 1,13 & 0,3 & 90 vs. 85 & 1,00 \\
\hline & $\leq 85$ & 5228 & $169 / 105$ & $141 / 83$ & 4,1 & 85 vs. 80 & 1,07 & 0,3 & 85 vs. 80 & 0,86 \\
\hline & $\leq 80$ & 5237 & $169 / 105$ & $139 / 81$ & 3,9 & 90 vs. 80 & 1,21 & 0,4 & 90 vs. 80 & 0,85 \\
\hline \multirow[t]{3}{*}{ Con $\mathrm{ECl}$} & $\leq 90$ & 1019 & $174 / 106$ & $146 / 85$ & 9,3 & 90 vs. 85 & 1,37 & 1,0 & 90 vs. 85 & 1,36 \\
\hline & $\leq 85$ & 1036 & 173/106 & $144 / 83$ & 6,8 & 85 vs. 80 & 0,82 & 0,8 & 85 vs. 80 & 1,46 \\
\hline & $\leq 80$ & 1025 & $173 / 106$ & $143 / 81$ & 8,3 & 90 vs. 80 & 1,12 & 0,5 & 90 vs. 80 & 1,99 \\
\hline
\end{tabular}

$\mathrm{IM}=$ infarto de miocardio; $\mathrm{PS}$ = presión sistólica; $\mathrm{PD}$ = presión diastólica; $\mathrm{RR}$ = riesgo relativo; pac. = paciente.

en $51 \%$ de eventos cardiovasculares en comparación a aquellos con $\mathrm{PD}<90$ $\mathrm{mmHg}{ }^{(18)}$. Sin embargo, en este estudio se omitió inicialmente datos relacionados a la presencia de la curva J, al referir que hubo problemas de espacio con la editorial, pero que fueron posteriormente publicados por Cruickshank ${ }^{(19)}$ y que se muestra en la tabla 1. Esta información relevante que precisamente compara pacientes con cardiopatía isquémica y no isquémica muestra la presencia de una curva J con eventos coronarios, pero no con stroke. Para este análisis se incluyeron 3080 pacientes con enfermedad cardiaca isquémica, en los que había solo 286 pacientes con antecedente de infarto de miocardio. Se describió un incremento en $22 \%$ de riesgo de infarto de miocardio si la PD se encontraba en el grupo de tratamiento $<80 \mathrm{mmHg}$, en comparación a si se encontraba en el grupo $<85 \mathrm{mmHg}$, por lo que una excesiva reducción de la presión diastólica en la población con cardiopatía isquémica puede ser insegura. Por otro lado, se determinó además que en la población no diabética no se evidenció un beneficio significativamente estadístico en disminuir la presión en menos de $90 \mathrm{mmHg}$.

Considerando el riesgo asociado que puede existir en los pacientes con enfermedad cardiovascular isquémica, nos preguntamos si debemos de insistir en un tratamiento agresivo de la hipertensión arterial en los diferentes grupos poblacionales. El estudio FEVER ${ }^{(20)}$ realizado en más de 9000 pacientes señala un beneficio de reducción de eventos cardiovascu- lares con solo una discreta reducción de PA (de 154/91 a 137/82 mmHg).

En los pacientes con diabetes mellitus, en diferentes comités, se recomienda una presión objetivo de $<130 / 80 \mathrm{mmHg}$, lo cual devino de una recomendación inicial en relación a estudios como el HOT. Sin embargo, hemos apreciado que en el mismo estudio HOT se describe una curva J en pacientes con enfermedad cardiaca isquémica. El estudio ACCORD ${ }^{(21)}$ de reciente publicación incluyó más de 10000 pacientes, en el cual se observó que aquellos pacientes sometidos a un tratamiento intensivo con una PAS $<120$ $\mathrm{mmHg}$ y $\mathrm{HbA} 1 \mathrm{c}<6 \%$ tuvieron incremento en su mortalidad total y cardiovascular, por lo que el estudio tuvo que ser terminado precozmente. En un análisis post hoc del estudio IDNT ${ }^{(22)}$ (que incluía pacientes diabéticos con niveles de creatinina hasta $3 \mathrm{mg} / \mathrm{dL}$ y proteinuria $>900 \mathrm{mg} /$ día), se describió que una $\mathrm{PD}<85 \mathrm{mmHg}$ se asocia a mayor mortalidad e infarto de miocardio. Sin embargo, esto a su vez requiere considerar el factor relacionado a la proteinuria y función renal. Otros estudios, como el estudio $\mathrm{ABCD}$, precisa que un tratamiento agresivo reduce la incidencia de complicaciones micro y macrovasculares, en donde una PD de $75 \mathrm{mmHg}$ no se asocia a riesgo cardiovascular. Sin embargo, este objetivo fue estudiado en pacientes normotensos con presiones diastólicas basales de 80 a $89 \mathrm{mmHg}$, y que en la terapia intensiva tuvo una reducción promedio de PD en $75 \mathrm{mmHg}$. Considerando que no se tiene aun suficiente evidencia para una reco- mendación precisa, las guías europeas ${ }^{(23)}$ de hipertensión arterial, en su recomendación vigente del 2009 no recomiendan un valor objetivo de $\mathrm{PA}<130 / 80 \mathrm{mmHg}$ en el paciente diabético.

En los pacientes con enfermedad renal crónica (ERC) se recomienda alcanzar una PA objetivo de $<130 / 80 \mathrm{mmHg}$, según guías DOQI ${ }^{(24)}$, en la cual se señala una evidencia tipo B, y en la que este concepto ha sido traspolado del paciente de riesgo alto, como el diabético, con el fin de evitar eventos cardiovasculares. Tampoco constituye una recomendación suficiente para las guías europeas de hipertensión arterial.

Otra recomendación conocida, de una PA objetivo de $<125 / 75 \mathrm{mmHg}$ en aquellos pacientes con ERC y proteinuria $>1$ $\mathrm{g} / \mathrm{día}$, se basa principalmente en el estudio MDRD, que fue un estudio en 840 pacientes, cuyas depuraciones de creatinina estaban entre 13 y $55 \mathrm{~mL} / \mathrm{min} / 1,73 \mathrm{~m}^{2}$, En su análisis secundario ${ }^{(25,26)}$, en relación al efecto de presión arterial, se observó que una $\mathrm{PA}<125 / 75 \mathrm{mmHg}$ reducía el progreso de ERC. Es importante señalar que en este estudio los pacientes tenían una PA basal de $130 / 80 \mathrm{mmHg}$, y existió una diferencia importante en relación a los resultados del estudio AASK ${ }^{(27)}$, estudio en el que pese a que existió una mayor reducción de la PA $(5 \mathrm{mmHg}$ del AASK versus $4 \mathrm{mmHg}$ del MDRD), no se reportó un beneficio significativo en la progresión de la enfermedad renal. Solo fue beneficioso el efecto antiproteinúrico del ramipril utilizado. 
En otros grupos de tratamiento, como el de los pacientes ancianos, también encontraremos algunas contradicciones, en donde además es notable que la mayoría de estudios ha reclutado pacientes con PAS $>160 \mathrm{mmHg}$; por lo que las recomendaciones actuales sobre el inicio de tratamiento en esta población se basan en estudios pos hoc. Bajo esta premisa, las guías europeas recomiendan en general una PAS objetivo de 130 a $139 \mathrm{mmHg}$ y PAD de 80 a $85 \mathrm{mmHg}$. Considerando la presencia de la curva J, en especial en la población con cardiopatía isquémica, que a su vez es prevalente en pacientes diabéticos y nefrópatas, constituye al momento una opción valida. Aún no es posible establecer un punto $\mathrm{J}$ nadir a partir del cual precisaremos aumento del riesgo cardiovascular. Sin embargo, debemos tener en cuenta la presencia de la curva según los diferentes reportes cuando la PA es menor de 120/80. Este debe ser nuestro menester para un óptimo control de la presión arterial.

\section{REFERENCIAS BIBLIOGRÁFICAS}

1. JNC 7, Seventh report of the Joint National Committee on Prevention, Detection, Evaluation, and Treatment of High Blood Pressure. Hypertension. 2003;42:1206-52.

2. Williams B, Poulter NR, Brown MJ, Davis M, Mclnnes GT, Potter JF, Sever PS, Thom SM; BHS guidelines working party, for the British Hypertension Society. British Hypertension Society guidelines for hypertension management 2004 (BHS-IV): summary. BMJ. 2004;328(7440):634-40.

3. Stewart IM. Relation of reduction in pressure to first myocardial infarction in patients receiving treatment for severe hypertension. Lancet. 1979;1(8121):861-5.

4. Franklin SS. Isolated systolic hypertensión and the J curve of cardiovascular disease risk. Artery Res. 2010;4:1-6.

5. Messerli FH, Panjrath GS. The J curve between blood pressure and coronary artery disease or essential hypertension. J Am Coll Cardiol. 2009;54:1827-34.

6. Kannel WB, Wilson PW, Nam BH, D'Agostino $R B$, Li J. A likely explanation for the J curve of blood pressure cardiovascular risk. Am I Cardiol. 2004;94(3):380-4.
7. Yoon SS, Ostchega Y, Louis T. NCHS Data Brief. Recent trends in the prevalence of high blood pressure and its treatment and control. Disponible en: http://www.cdc.gov/nchs/data/databriefs/ db48.htm

8. Thune JJ, Signorovitch J, Kober L, Velazquez EJ, McMurray JJ, Califf RM, Maggioni AP, Rouleau JL, Howlett J, Zelenkofske S, Pfeffer MA, Solomon SD. Effect of antecedent hypertension and follow-up blood pressure on outcomes after high-risk myocardial infarction. Hypertension. 2008;51(1):48-54.

9. Messerli FH, Mancia G, Conti CR, Hewkin AC, Kupfer S, Champion A, Kolloch R, Benetos A, Pepine CJ. Dogma disputed: can aggressively lowering blood pressure in hypertensive patients with coronary artery disease be dangerous? Ann Intern Med. 2006;144(12): 884-93.

10. Polese A, De Cesare N, Montorsi P, Fabbiocchi F, Guazzi M, Loaldi A, Guazzi MD. Upward shift of the lower range of coronary flow autoregulation in hypertensive patients with hypertrophy of the left ventricle. Circulation. 1991;83(3):845-53.

11. Farnett L, Mulrow CD, Linn WD, Lucey CR, Tuley $M R$. The J curve phenomenon and the treatment of hypertension. JAMA. 1991;265(4):489-95.

12. MacMahon S, Peto R, Collins R, Godwin J, MacMahon S, Cutler J, Sorlie P, Abbott R, Collins R, Neaton J, Abbott R, Dyer A, Stamler J. Blood pressure, stroke, and coronary heart disease: Part 1, prolonged differences in blood pressure: prospective observational studies corrected for the regression dilution bias. Lancet 1990;335:76574.

13. Fletcher AE, Bulpitt CJ. How far should blood pressure be lowered? NEJM. 1992;326(4):2514.

14. Vaccarino V, Berger AK, Abramson J, Black HR, Setaro JF, Davey JA, Krumholz HM. Pulse pressure and risk of cardiovascular events in the systolic hypertension in the elderly program. Am J Cardiol. 2001;88(9):980-6.

15. Somes GW, Pahor M, Shorr RI, Cushman WC, Applegate WB. The role of diastolic blood pressure when treating isolated systolic hypertension. Arch Intern Med. 1999;159(17):2004-9.

16. Prospective Studies Collaboration, Age-specific relevance of usual blood pressure to vascular mortality: a meta-analysis of individual data for one million adults in 61 prospective studies. Lancet. 2002;360:1903-13.

17. Hanson L, Zanchetti A, Carruthers SG, Dahlöf B, Elmfeldt D, Julius S, Ménard J, Rahn KH, Wedel H, Westerling S. Effects of intensive blood-pressure lowering and low-dose aspirin in patients with hypertension: principal results of the Hypertension
Optimal Treatment (HOT) randomised trial. Lancet. 1998;351(9118):1755-62.

18. Hanson L. Antihypertensive treatment: Does the J curve exist? Cardiovasc Drugs Ther. 2000;14(4):367-72.

19. Cruickshank JM. Antihypertensive treatment and the J curve. Cardiovasc Drugs Ther. 2000;14(4):373-9.

20. Liu L. The felodipine event reduction (FEVER) Study: a randomized long-term placebo-controlled trial in Chinese hypertensive patients. J Hypertension. 2005;23(12): 2157-72.

21. ACCORD Study group, Cushman WC, Evans GW, Byington RP, Goff DC Jr, et al. Effects of intensive blood pressure control in type 2 diabetes mellitus. NEJM. 2010;362(17): 1575-85.

22. Berl T, Hunsicker LG, Lewis JB, Pfeffer MA, Porush $J G$, Rouleau JL, Drury PL, Impact of achieved blood pressure on cardiovascular outcomes in the Irbesartan Diabetic Nephropathy trial, J Am Soc Nephrol. 2005;16(7):2170-9.

23. Reappraisal of European Guidelines on hypertension management: a European Society of Hypertension Task Force document. J Hypertension. 2009;27(119):2121-58.

24. ALLHAT: still providing correct answers after 7 years. Curr Opin Cardiol. 2010:25(4):355-65.

25. Lazarus JM, for the Modification of Diet in Renal Disease Study Group. Achievement and safety of a low blood pressure goal in chronic renal disease. Hypertension. 1997;29:641-50.

26. Sarna MJ, Greene T, Wang X, Beck G, Kusek JW, Collins AJ, Levey AS. The effect of a lower target blood pressure on the progression of kidney disease: long-term follow-up of the modification of diet in renal disease study. Ann Intern Med. 2005;142(5):342-51.

27. Agodoa L, Appel L, Bakris GL, et al; for the African American Study of Kidney Disease and Hypertension (AASK) Study Group Effect of ramipril vs amlodipine on renal outcomes in hypertensive nephrosclerosis: a randomized controlled trial. JAMA. 2001;285(21):2719-28.

Conflicto de intereses: Ninguno

Correspondencia:

Dr. Edwin Castillo Velarde

Servicio de Nefrología, Hospital Nacional Guillermo Almenara

Av. Grau 800, Lima, Perú

Teléfono: 051-13242983

Correo electrónico: edwin_castillo2002@yahoo.es 